\title{
Optimized synthesis of ultrahigh-surface-area and oxygen-doped carbon nanobelts for high
}

\section{cycle-stability lithium-sulfur batteries}

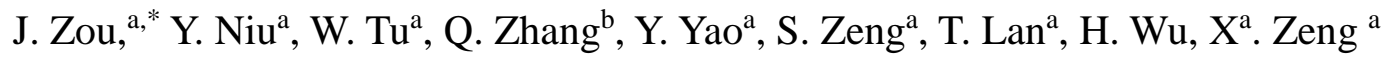

a Shenzhen Key Laboratory of Special Functional Materials \& Shenzhen Engineering Laboratory for Advance Technology of Ceramics, College of Materials Science and Engineering, Shenzhen University, Shenzhen 518060, P. R. China.

${ }^{\mathrm{b}}$ School of Aerospace, Transport and Manufacturing, Cranfield University, Cranfield, Bedfordshire, MK43 OAL, UK.

* Corresponding author: J.Zou

E-mail address: zoujizhao@szu.edu.cn

\begin{abstract}
:
Hierarchical clews of carbon nanobelts (CsCNBs) with ultrahigh specific surface area $\left(2300 \mathrm{~m}^{2}\right.$ $\mathrm{g}^{-1}$ ) and large pore volume (up to $1.29 \mathrm{~cm}^{3} \mathrm{~g}^{-1}$ ) has been successfully fabricated through carbonization and $\mathrm{KOH}$ activation of phenolic resin based nanobelts. The product possesses hierarchically porous structure, three-dimensional conductive network framework, and polar oxygen-rich groups, which are very befitting to load sulfur leading to excellent cycling stability of lithium-sulfur batteries. The composites of $\mathrm{CsCNBs} /$ sulfur exhibit an ultrahigh initial discharge capacity of $1245 \mathrm{~mA} \mathrm{~h} \mathrm{~g}^{-1}$ and ultralow capacity decay rate as low as $0.162 \%$ per cycle after 200 cycles at $0.1 \mathrm{C}$. Even at high current rate of $4 \mathrm{C}$, the cells still display a high initial discharge capacity $\left(621 \mathrm{~mA} \mathrm{~h} \mathrm{~g}^{-1}\right)$ and ultralow capacity decay rate (only $0.039 \%$ per cycle) after 1000 cycles. These encouraging results indicate that polar oxygen-containing functional groups are important for improving the electrochemical performance of carbons. The oxygen-doped carbon nanobelts have excellent energy storage potential in the field of energy storage.
\end{abstract}




\section{Introduction}

Lithium batteries are widely used in energy storage power systems such as hydropower, firepower, wind power and solar power plants, electric bicycles, aerospace and many other fields. [1-2]. However, the traditional Lithium batteries cannot satisfy the high requirement of more integrated devices with high energy-consumption as well as the environmental protection concept [3-5]. Lithium-sulfur batteries (LiSBs) are notable for their ultrahigh theoretical energy density (2600 $\mathrm{W} \mathrm{h} \mathrm{kg}^{-1}$ ) that doubles the energy density of lithium-ion batteries [6-8], cheap raw materials and little pollution to the environment [9-10]. Therefore, LiSBs are very promising batteries for energy storage applications. However, there are three major drawbacks for LiSBs which hamper their development in energy storage fields. First, elemental sulfur has poor electrical conductivity, and final reaction products $\left(\mathrm{Li}_{2} \mathrm{~S}_{2}\right.$ and $\left.\mathrm{Li}_{2} \mathrm{~S}\right)$ are also electronic insulators, which are not beneficial to the high-rate performance of batteries [11-14]. Second, lithium polysulfide compounds $\left(\mathrm{Li}_{2} \mathrm{~S}_{8}\right.$, $\mathrm{Li}_{2} \mathrm{~S}_{6}$ and $\mathrm{Li}_{2} \mathrm{~S}_{4}$ ) are easily soluble in the electrolyte, resulting in a shuttle effect which easily destroys the electrolyte interface film (SEI film) $[12,15]$. Third, sulfur will undergo a volumetric expansion of $\sim 80 \%$ during charging and discharging process, which causes the pulverization of the electrode materials and thus damages the batteries [16-17].

In recent ten years, in order to solve the problems in LiSBs, researchers have conducted a lot of research on the modification of composite cathode materials. One of the most important, porous carbons have received great attention and research for its high electrical conductivity, high specific surface area (SSA) [18], high porosity, high chemical and mechanical stability, and ease of preparation. According to its structure, porous carbon is divided into one-dimensional materials (such as carbon nanotubes), two-dimensional materials (such as graphene) and three-dimensional materials (such as hollow carbon spheres). In a variety of porous carbon, hierarchical porous carbons (HPCs) have been more widely researched due to the co-existence of micropore $(<2 \mathrm{~nm})$, 
mesopore $(2-50 \mathrm{~nm})$, and macropore (> $50 \mathrm{~nm})$ [19-22]. Hierarchical pores can make a synergistic effect of in LiSBs. Micropores provide anchoring sites for binding lithium polysulfide. Mesopores are commonly used to enhance the physical properties of microporous or macroporous networks. The smaller mesopores along with the micropores store sulfur and adsorb polysulfides, further slowing down the decay of capacity. The larger mesopores along with macropores promote charge transport and ensure electrolyte infiltration, which thereby improves sulfur utilization of active materials. The macroporous network serves to contain and transport the electrolyte to ensure good electrolyte impregnation. The physisorption characteristic of the pores can effectively improve the cycling stability of LiSBs.

In addition, Cui groups [15, 23] studied the interaction and binding energies of various functional groups (such as carbon groups and oxygen-rich groups) and lithium sulfide (LiS) species using density functional theory based on the vinyl polymer. As a result, carbon-based groups, such as alkanes, demonstrate lower binding energy with LiS species $(0.30 \mathrm{eV})$ and oxygen-rich groups containing esters, amides, ketones and ethers, demonstrate higher binding energies between 1.01 and $1.26 \mathrm{eV}$. Oxygen-containing functional groups have been found to improve the combination with lithium polysulfides. In short, the chemisorption between LiS species and oxygen-rich groups has a potential improvement effect on the cycle-stability of LiSBs. Cheng and colleagues [24] found that the strong chemical combination between sulfur and oxygen-containing functional groups (mainly $-\mathrm{OH}$ and $\mathrm{C}=\mathrm{O}$ ) can significantly reduce the dissolution/diffusion of lithium polysulfides on reduced graphene oxide. Further, the cycle stability of the lithium-sulfur battery is improved.

$\mathrm{KOH}$ activation is a very promising method for the preparation of HPCs, because of its low activation temperature, high carbon yield, well-defined pore size distribution and ultrahigh specific surface area [18]. Using biomass waste (soybean residue) as precursor, Zhou et al. [25] successfully prepared a new type of honeycomb nitrogen-oxygen co-doped porous carbon using $\mathrm{KOH}$ activation. 
The derived porous carbon has a special multi-stage pore structure, a large specific surface area (SSA) $\left(2690.3 \mathrm{~m}^{2} \mathrm{~g}^{-1}\right)$, a high pore volume $\left(1.34 \mathrm{~cm}^{3} \mathrm{~g}^{-1}\right)$, and a suitable $\mathrm{N}$, O co-doping. The carbon/sulfur composite [26-31] prepared by the porous carbon (sulfur content of $64.5 \%$ ) exhibits an excellent cycle stability in LiSBs, and the capacity decay rate after cycling 600 times at a current rate of $1 \mathrm{C}$ is as low as $0.063 \%$ per cycle.

Therefore, we prepared the hierarchical clews of carbon nanobelts (CsCNBs) by $\mathrm{KOH}$ activation for LiSBs (Scheme 1) in this work. $\mathrm{KOH}$ activation is employed to increase the SSA, pore volume, and number of polar oxygen-containing functional groups. By controlling the mass ratio of $\mathrm{KOH} / \mathrm{C}$, the CsCNBs-800*4 has obtained the highest SSA of $2300 \mathrm{~m}^{2} \mathrm{~g}^{-1}$ and the biggest pore volume of $1.29 \mathrm{~cm}^{3} \mathrm{~g}^{-1}$. Fundamentally, the CsCNBs with hierarchically porous structure, three-dimensional conductive network framework, and polar oxygen-rich groups are very befitting to load sulfur for the improvement of cycle-stability of LiSBs. Hence, CsCNBs/S composites were prepared. CsCNBs-800*4/S cells have an ultrahigh capacity at low current rates. The initial discharge/charge capacity is $1245 / 1187 \mathrm{~mA} \mathrm{~h} \mathrm{~g}^{-1}$ at $0.1 \mathrm{C}$. The capacity decay rate is as low as $0.162 \%$ per cycle for 200 cycles (voltage window is $1.8-2.8 \mathrm{~V}$ ). At high current rates (>xxxC), the cells still exhibit an excellent cycle stability. At $4 \mathrm{C}$, the initial discharge/charge capacity is $621 / 518$ $\mathrm{mA} \mathrm{h} \mathrm{g}^{-1}$. After 1000 cycles, the capacity decay rate (only $0.039 \%$ per cycle) is extremely low. 


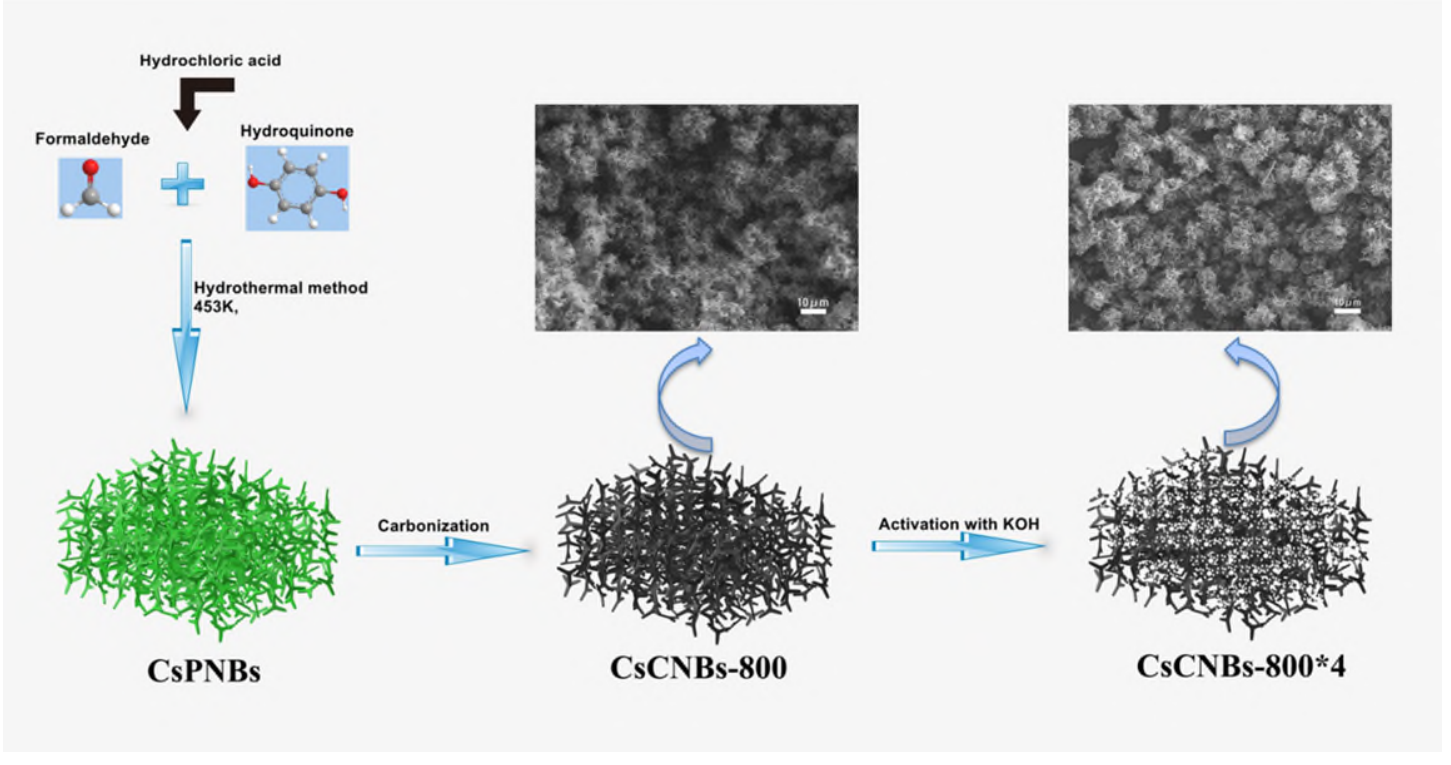

Scheme 1. A schematic illustration of the preparation of carbon nanobelts.

\section{Experimental section}

\section{Materials}

Hydroquinone [Analytical reagent (AR), 99\%], formaldehyde (AR, $37 \mathrm{wt} \%$ ), hydrochloric acid (AR, 37wt\%), sulfur (AR, 99.9\%), $\mathrm{CS}_{2}(\mathrm{AR}), \mathrm{KOH}(\mathrm{GR}, 95 \%)$ and tetrahydrofuran (THF, AR) were purchased from Shanghai Macklin Company (P. R. China).

\section{Synthesis of CsPNBs}

CsPNBs were synthesized according to ref. 22. In general, $1.65 \mathrm{~g}$ of hydroquinone, $2.5 \mathrm{~mL}$ of $37 \mathrm{wt} \%$ formaldehyde and $115 \mathrm{~mL}$ of $10 \mathrm{wt} \%$ hydrochloric acid are thoroughly mixed in a $200 \mathrm{~mL}$ autoclave with a polytetrafluoroethylene liner. Then, the autoclave was sealed and heated in an oven at $180{ }^{\circ} \mathrm{C}$ for 12 hours. The resulting black spongy product was filtered and washed with deionized water. The filter cake was dried under vacuum at $60{ }^{\circ} \mathrm{C}$ for $6 \mathrm{~h}$. Finally, $2.0 \mathrm{~g}$ of light and dark brown powder was collected. The yield is close to the quantitative value (based on hydroquinone).

\section{Fabrication of CsCNBs}

CsPNBs $\left(1.80 \mathrm{~g}\right.$ ) was heated to $800{ }^{\circ} \mathrm{C}$ (with a heating rate of $5{ }^{\circ} \mathrm{C} \mathrm{min}^{-1}$ ) under a flow of argon $\left(50 \mathrm{~mL} \mathrm{~min}{ }^{-1}\right)$ and then cooled down. This sample was called CsCNBs-800. CsCNBs-800 was chemically activated with $\mathrm{KOH}$. The mixture, consisting of CsCNBs-800 and a certain amount of 
$\mathrm{KOH}$, was activated under a flow of argon $\left(50 \mathrm{~mL} \mathrm{~min}^{-1}\right)$ at $400{ }^{\circ} \mathrm{C}$ for $0.5 \mathrm{~h}$ and then at $800{ }^{\circ} \mathrm{C}$ for $1 \mathrm{~h}$ at a heating rate of $5{ }^{\circ} \mathrm{C} \mathrm{min}^{-1}$. The weight ratios of $\mathrm{KOH} / \mathrm{C}$ were 3,4 and 5 . Therefore, the samples were denoted according to the mass ratio as CsCNBs- $800 * 3$, CsCNBs- $800 * 4$ and CsCNBs-800*5, respectively. CsCNBs-950-40 was physically activated with $\mathrm{CO}_{2}$ according to ref.

22. The activation time was $40 \mathrm{~min}$.

\section{Synthesis of $\mathrm{CsCNBs/S}$ composites}

CsCNBs $(0.08 \mathrm{~g})$ and sulfur $(0.12 \mathrm{~g})$ were dispersed in $\mathrm{CS}_{2}(1 \mathrm{ml})$ and stirred till totally dry. To ensure a complete infiltration of sulfur into CsCNBs, the mixture was heated at $155{ }^{\circ} \mathrm{C}$ for $12 \mathrm{~h}$. Therefore, all composites contain CsCNBs and sulfur are denoted as CsCNBs-800/S, CsCNBs-800*3/S, CsCNBs-800*4/S, CsCNBs-800*5/S and CsCNBs-950-40/S. Meanwhile, the mass fraction of sulfur in each composite was $60 \%$. Similarly, composite (90 wt $\%$ of sulfur) was also prepared and named CsCNBs-800*4/S90.

\section{Characterization}

Field emission scanning electron microscopy (FESEM) images were collected via a field emission SU-70 microscope. High-resolution transmission electron microscopy (HRTEM) images were obtained with a JEOL JEM2010 electron microscope. High-resolution X-ray diffraction (HR-XRD) patterns were recorded on a Bruker D8 Advance diffractometer using $\mathrm{Cu}-\mathrm{K} \alpha$ radiation (40 kV and $200 \mathrm{~mA}$ ). Raman spectra were measured using a laser confocal Raman spectrometer quipped with an argon-ion laser $(514.5 \mathrm{~nm})$. Measurement of the Brunauer-Emmett-Teller (BET) specific surface areas was performed at $77 \mathrm{~K}$ using a low-temperature nitrogen adsorption surface area analyser (ASAP 2020, Micromeritics Ins, USA). The total pore volume and pore size distribution were calculated by a density functional theory (DFT) model. Thermogravimetric analysis (TGA) was performed on a TGA-Q55 thermal gravimetric analyzer. X-ray photoelectron spectroscopy (XPS) experiments were carried out on a Microlab 350 spectrometer using a 
monochromatic Mg-Ka X-ray source.

\section{Cell fabrication and measurements}

According to the Ref. 22, the lithium-sulfur cells (CR2016) were fabricated in a glove box $(\mathrm{O} 2<0.1 \mathrm{ppm} ; \mathrm{H} 2 \mathrm{O}<0.1 \mathrm{ppm})$ to test the electrochemical performances of $\mathrm{CsCNBs} / \mathrm{S}$. The cathode slurry was prepared by mixing $80 \%$ of the composite, $10 \%$ Super-P and $10 \%$ of PVDF binder in 1-methyl-2-pyrrolidinone (NMP). The slurry was blade cast onto carbon-coated aluminum foil and dried at $60{ }^{\circ} \mathrm{C}$ for $12 \mathrm{~h}$ under vacuum. For composites with $60 \%$ sulfur, the sulfur loading for each cathode foil was about $1.0 \mathrm{mg} \mathrm{cm} \mathrm{cm}^{-2}$. The sulfur loading for CsCNBs-800*4/S90 was approximately $1.8 \mathrm{mg} \mathrm{cm}^{-2}$. The diameter of the cathode was $15 \mathrm{~mm}$. The electrolyte consisted of $1 \mathrm{M}$ LiTFSI and $0.1 \mathrm{M} \mathrm{LiNO}_{3}$ in DME/DOL (1,2-dimethoxyethane/1,3-dioxolane)(1:1 by volume). The ratio of electrolyte to sulfur was $20 \mu \mathrm{L} \mathrm{mg}^{-1}$. The cyclic voltammetry (CV) measurements were carried out on an electrochemical workstation (CHI660E, Chenhua Instrument Company, Shanghai, China) over the potential range of $1.8-2.8 \mathrm{~V}$ vs. $\mathrm{Li}^{+} / \mathrm{Li}$ at a scan rate of $0.2 \mathrm{mV} \mathrm{s}^{-1}$. The charge/discharge experiments were conducted on NEWARE (Shenzhen, China) instruments (model 5 V-10 mA and 5 $\mathrm{V}-50 \mathrm{~mA}$ ) with a voltage window of $1.8-2.8 \mathrm{~V}$ vs. $\mathrm{Li}^{+} / \mathrm{Li}$.

\section{Results and discussion}

The FESEM images of carbonized sample (CsCNBs-800) and activated samples (CsCNBs-800*4 and CsCNBs-950-40) obtained by different methods are shown in Fig. 1. As shown in Fig. 1a, 1c and 1e, it can be clearly seen that the diameter of clews is in the range of 5-30 $\mu \mathrm{m}$. Meanwhile, there is no obvious change in the morphology of clews after activation. In Fig. 1b, $1 \mathrm{~d}$ and $1 \mathrm{f}$, it can be found that the clews are disorderly intertwined by a large number of very thin nanobelts. In addition, the nanobelt width of the activated samples is narrower than that of the carbonized sample. 

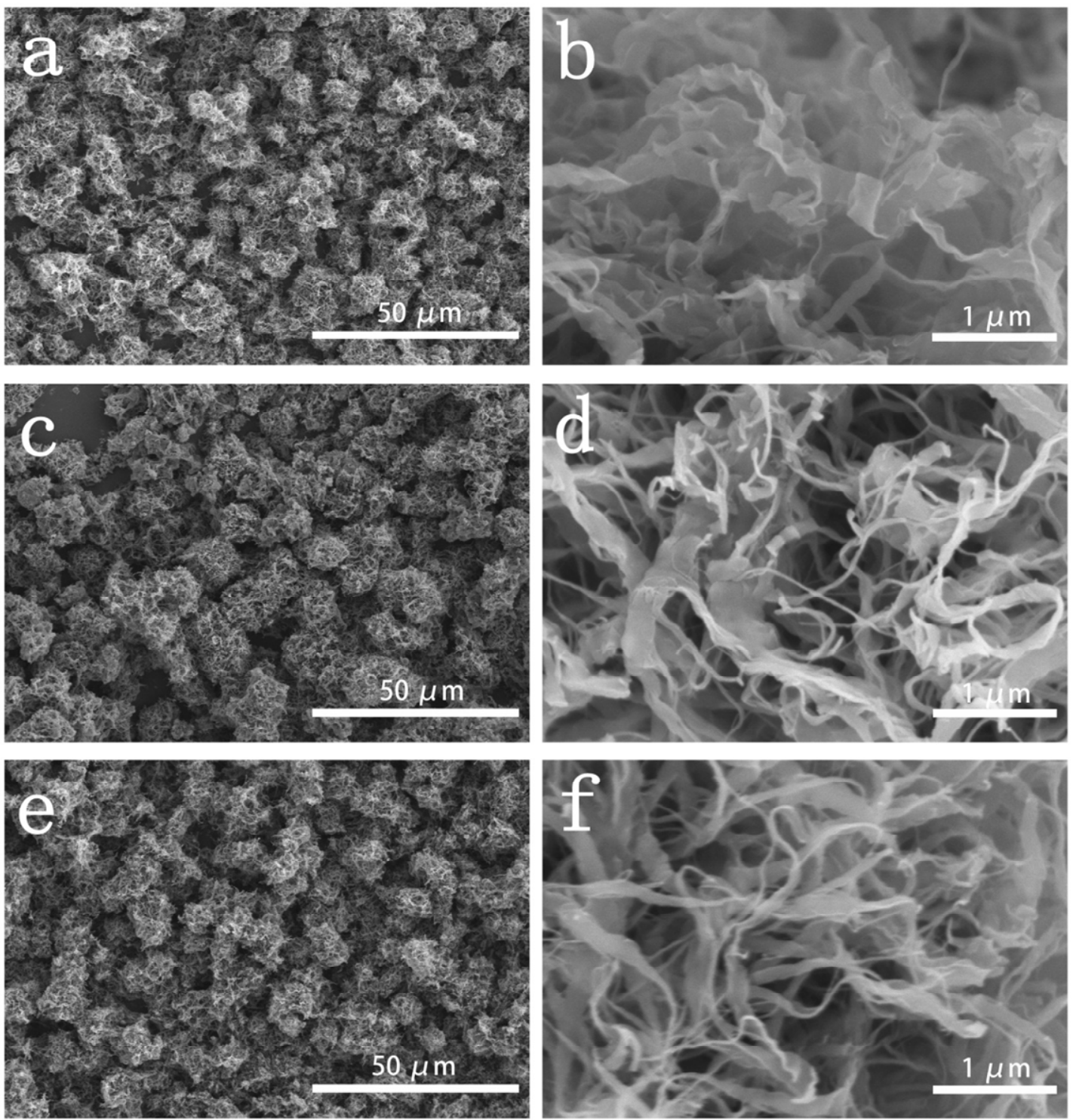

Fig. 1. FESEM images of (a, b) CsCNBs-800, (c, d) CsCNBs-800*4, (e, f) CsCNBs-950-40.

The HRTEM images of CsCNBs-800, CsCNBs-800*4 and CsCNBs-950-40 samples are shown in Fig. 2. In Fig. 2a, 2c and 2e, it can be clearly observed that three samples display same structure, which indicates that the nanobelts remain unchanged after carbonization and activation. It can be seen that the nanobelts are narrow and thin, consistent with the FESEM images. In Fig. 2b, 2d and $2 \mathrm{f}$, there are a lot of disordered black stripes, which correspond to the (002) facet of amorphous carbon [32-34]. High density white spots can also be seen, which are mainly characterized by mesopores. 

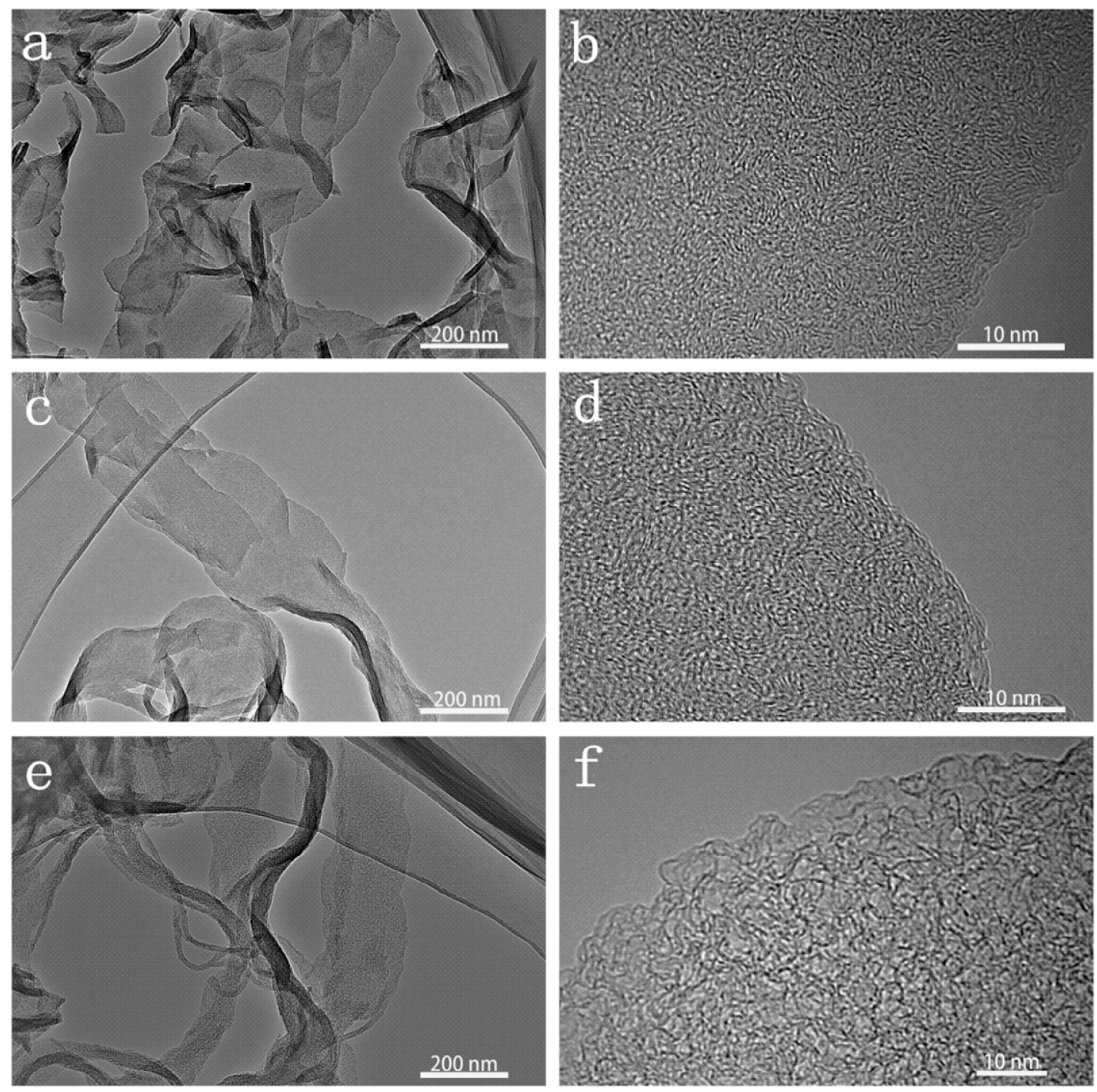

Fig. 2. HRTEM images of (a, b) CsCNBs-800, (c, d) CsCNBs-800*4, (e, f) CsCNBs-950-40.

Fig. 3a shows the XRD patterns of carbonized sample (CsCNBs-800) and activated samples (CsCNBs-800*3, CsCNBs-800*4, CsCNBs-800*5 and CsCNBs-950-40), which exhibit two diffraction peaks like a steamed bread at approximately $24^{\circ}$ and $43^{\circ}$ [35-36], corresponding to the diffraction of (002) and (100) planes of graphite, indicating that this microporous carbon consists of small domains of ordered graphene sheets, typical for amorphous carbons with non-crystalline structures [37-39]. After activation, the diffraction peaks of samples are much weaker, especially CsCNBs-950-40, indicating that activation is very effective in increasing disorder and defects. The Raman spectra of all samples are shown in Fig. 3b, which display two characteristic peaks unique to carbon at $1360 \mathrm{~cm}^{-1}$ and $1586 \mathrm{~cm}^{-1}$ [40]. The characteristic peak at $1586 \mathrm{~cm}^{-1}$ named $\mathrm{G}$ band is produced by the stretching movement of all $\mathrm{sp}^{2}$ atoms in the carboatomic rings or carbon chains. 
The characteristic peak at $1360 \mathrm{~cm}^{-1}$ named $\mathrm{D}$ band is generated by the breathing vibration mode of $\mathrm{sp}^{2}$ atoms in the hexagonal carbocycles, and induced by disorder and defects. Generally, the $\mathrm{R}$ value (intensity ratio of $\mathrm{I}_{\mathrm{D}} / \mathrm{I}_{\mathrm{G}}$ ) represents the degree of graphitization and disorder of carbons [41-43]. In comparison to CsCNBs-800, the $\mathrm{R}$ values of samples after activation are higher, especially CsCNBs-950-40, which is consistent with the analytical result of the XRD patterns. Especially, there is a $2 \mathrm{D}$ characteristic band at $2700 \mathrm{~cm}^{-1}$ in the Raman spectrum of samples after $\mathrm{KOH}$ activation, indicating the presence of few layer graphene [44-47]. Fig. 3c shows the nitrogen adsorption-desorption isotherms of CsCNBs-800, CsCNBs-800*3, CsCNBs-800*4, CsCNBs-800*5 and CsCNBs-950-40, which characterize the properties of the surface and pores. According to the IUPAC classification, the isotherm of all CsCNBs samples display the characteristic of type I/IV isotherms, indicating the existence of a large number of micropores and mesopores. At a relative pressure bigger than 0.9 , the degree of nitrogen adsorption isotherms rapidly rises, indicating that macropores are ubiquitous in all samples. The pore size distribution (PSD) curve based on the DFT theory also verifies this conclusion. In Fig. 3d, the PSD curve of CsCNBs-800 shows that micropores are mainly located at about $0.54,0.80$ and $1.18 \mathrm{~nm}$, and there is a small amount of mesoporous. The PSD curves of CsCNBs after activation show that mesopores are located at about $2.5 \mathrm{~nm}$ and micropores at $0.54,0.80,1.18$ and $1.48 \mathrm{~nm}$. The existence of numerous micropores and mesopores also confirms the results of HRTEM. The textural parameters of CsCNBs are shown in Table S1. Calculated by BET model, compared with other activated samples, CsCNBs-950-40 has a maximum SSA $\left(2610 \mathrm{~m}^{2} \mathrm{~g}^{-1}\right)$. However, the DFT approach is more accurate than the BET model to calculate the SSA for microporous carbon. For KOH activation, the SSA obtained by DFT approach increases with increasing degree of activation. When the $\mathrm{KOH} / \mathrm{C}$ mass ratio is 5 , the SSA is significantly decreased, indicating that the amount of $\mathrm{KOH}$ is excessive at this time. 
the same excellent characteristic parameters, such as, higher DFT specific surface area values and pore volume, and the rational distribution of micropores and mesopores, which are better than those of other samples. It is worth noting that the carbonization yield of CsCNBs-800*4 is 1.7 times that of CsCNBs-950-40. The inherent three-dimensional conductive network structure of the CsCNBs material ensures high accessibility and utilization of the nanobelt surface for ion adsorption. In addition, with the three-dimensional conductive network structure, the direct exposure of the highly porous nanobelt to the electrolyte can effectively shorten the transmission path and reduce the resistance of ion diffusion. These structural features can give CsCNBs an excellent potential for the uses in high performance LiSBs.
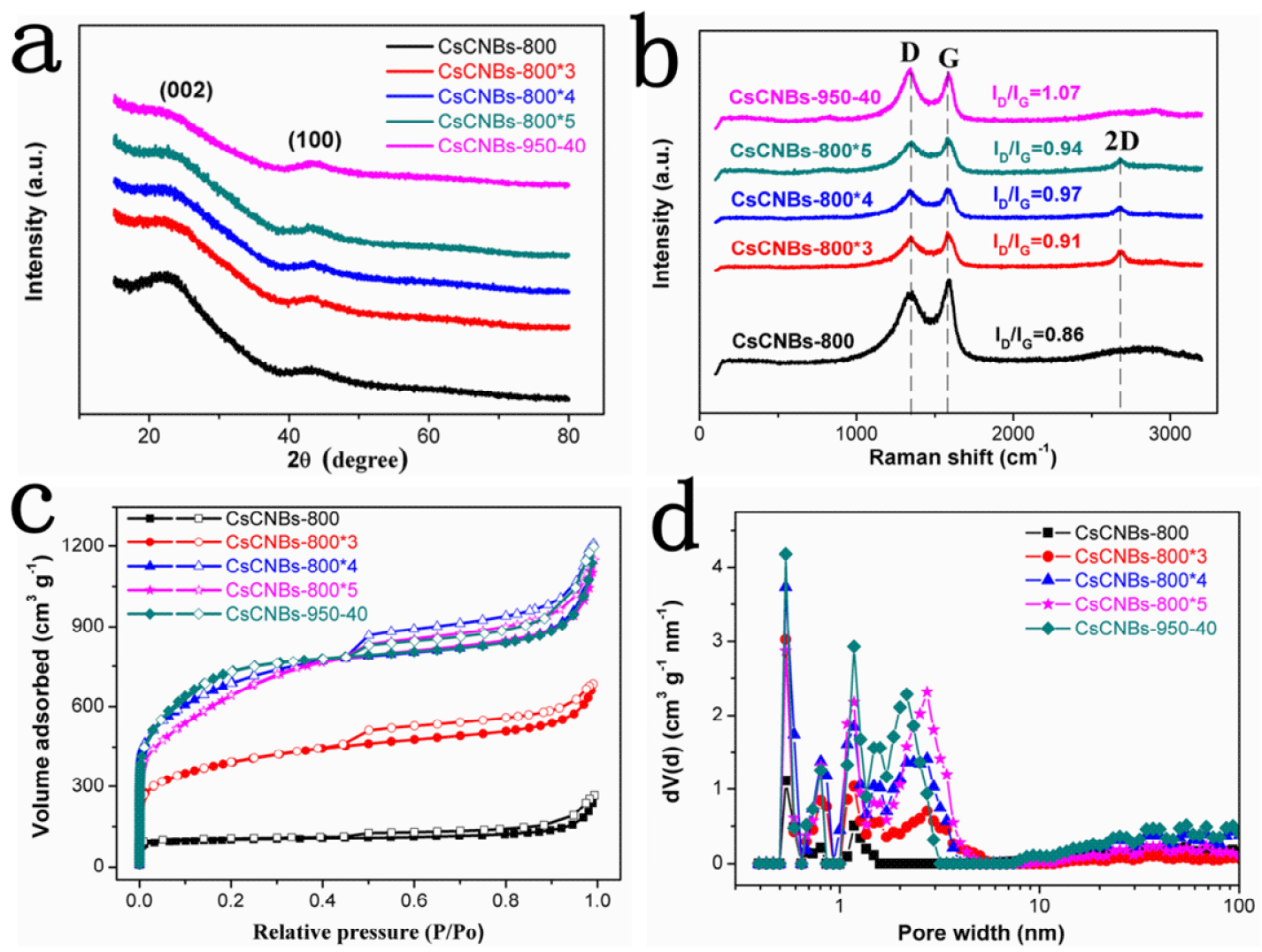

Fig. 3. (a) XRD patterns and (b) Raman spectra of the five carbon samples; (c) nitrogen adsorption-desorption isotherms and (d) PSD curves of the five carbon samples, calculated from the nitrogen adsorption isotherms using the DFT method.

The XPS survey spectra of CsCNBs-800, CsCNBs-800*4 and CsCNBs-950-40, including carbon and oxygen, are shown in Fig. 4. The carbon and oxygen contents are separately calculated from the peak areas of $\mathrm{C} 1 \mathrm{~s}$ and $\mathrm{O} 1 \mathrm{~s}$ peaks, which are consistent with the results of the elemental analysis test in Table S2. In Fig. 4a, 4d and 4g, the three samples possess the similar XPS survey 
spectra. In Fig. 4b, the C 1s spectrum of CsCNBs-800 sample displays three different component peaks, ascribed to $\mathrm{C}-\mathrm{C} / \mathrm{C}=\mathrm{C}(284.3 \mathrm{eV}), \mathrm{C}-\mathrm{O}(285.9 \mathrm{eV})$ and $\mathrm{C}=\mathrm{O}(287.2 \mathrm{eV})$. Correspondingly, the $\mathrm{O} 1 \mathrm{~s}$ spectrum of CsCNBs-800 clearly exhibits three oxygen-based groups, corresponding to $\mathrm{C}=\mathrm{O}(531.7 \mathrm{eV}), \mathrm{C}-\mathrm{O}(533.3 \mathrm{eV})$, and $\mathrm{H}_{2} \mathrm{O}(534.7 \mathrm{eV})$ in Fig. 4c. The spectra $(\mathrm{C}$ 1s spectrum in Fig. 4h and O 1s spectrum in Fig. 4i) of CsCNBs-950-40 sample is similar to CsCNBs-800 sample, excepting that the oxygen content is greatly reduced. After $\mathrm{KOH}$ activation, the $\mathrm{C} 1 \mathrm{~s}$ spectrum of CsCNBs-800*4 (Fig. 4e) has an extra weak peak ascribed to $\mathrm{O}-\mathrm{C}=\mathrm{O}$ species $(289.7 \mathrm{eV})$. The $\mathrm{O} 1 \mathrm{~s}$ spectrum of CsCNBs-800*4 (Fig. 4f) additionally possesses a considerable peak corresponding to $\mathrm{C}-\mathrm{OH} / \mathrm{C}-\mathrm{O}-\mathrm{C}(536.1 \mathrm{eV})$. The $\mathrm{C}-\mathrm{OH} / \mathrm{C}-\mathrm{O}-\mathrm{C}$ functional group (hydroxyl/epoxide) is a typical polar functional group with a high surface energy, which has a strong binding effect on polysulfides and is a great benefit to LiSBs that can achieve high-rate and high cycling stability. In general, compared to $\mathrm{CO}_{2}$ activation, $\mathrm{KOH}$ activation facilitates enhancing the retention of oxygen in carbons and creating a new polar oxygen-containing functional group.
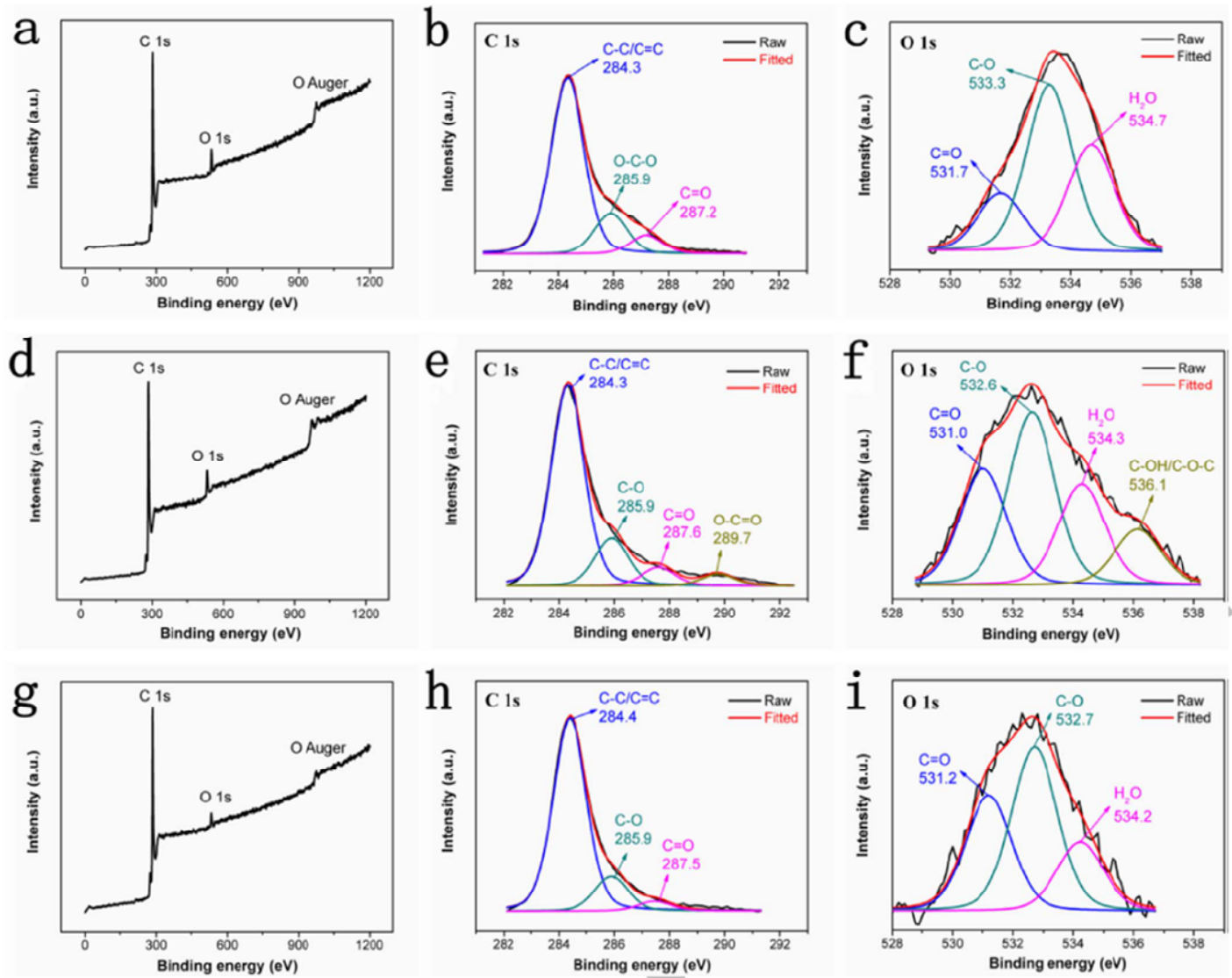
Fig. 4. XPS results of the CsCNBs-800 sample: (a) XPS survey, (b) $\mathrm{C} 1 \mathrm{~s}$ spectrum, and (c) O $1 \mathrm{~s}$ spectrum; XPS results of the CsCNBs-800*4 sample: (d) XPS survey, (e) C 1s spectrum, and (f) O 1s spectrum; XPS results of the CsCNBs-950-40 sample: (g) XPS survey, (h) C 1s spectrum, and (i) O 1s spectrum.

Due to its unique multi-level pore structure, three-dimensional high conductivity network, high surface area and pore volume, CsCNBs materials are expected to be used in various energy applications such as energy storage, adsorption and catalysis. As a proof of concept, CsCNBs are used for LiSBs after loading sulfur. For the consistency of the experimental variables, all CsCNBs samples were loaded with $60 \%$ sulfur. In order to study the electrochemical performance at high sulfur content, CsCNBs-800*4 is used to synthesize a carbon-sulfur composite (90 wt $\%$ of sulfur), due to its high specific surface area and high pore volume.

The FESEM images of all $\mathrm{CsCNBs} / \mathrm{S}$ samples are shown in Figure 5. As is illustrated in the picture, the nanobelts in CsCNBs-800/S and CsCNBs-800*3/S are thicker than other three samples with a sulfur content of $60 \%$, because there are some sulfur on the surface of nanobelts. However, CsCNBs-800*4/S, CsCNBs-800*5/S and CsCNBs-950-40/S have high pore volume, and almost all of the sulfur is adsorbed in the micropores and mesopores. As shown in Figure 5f, the sulfur is clearly coated on the surface of the CsCNBs-800*4/S90 nanobelts, which is attributed to the high pore volume of CsCNBs-800*4, whichcannot fully adsorb high sulfur content. In Fig. 6, the carbon, nitrogen, oxygen and sulfur mappings of CsCNBs-800*4/S display a similar pattern, demonstrating that the homogeneous distribution of these elements. It is further proved that the sulfur is well embedded in CsCNBs-800*4/S. 

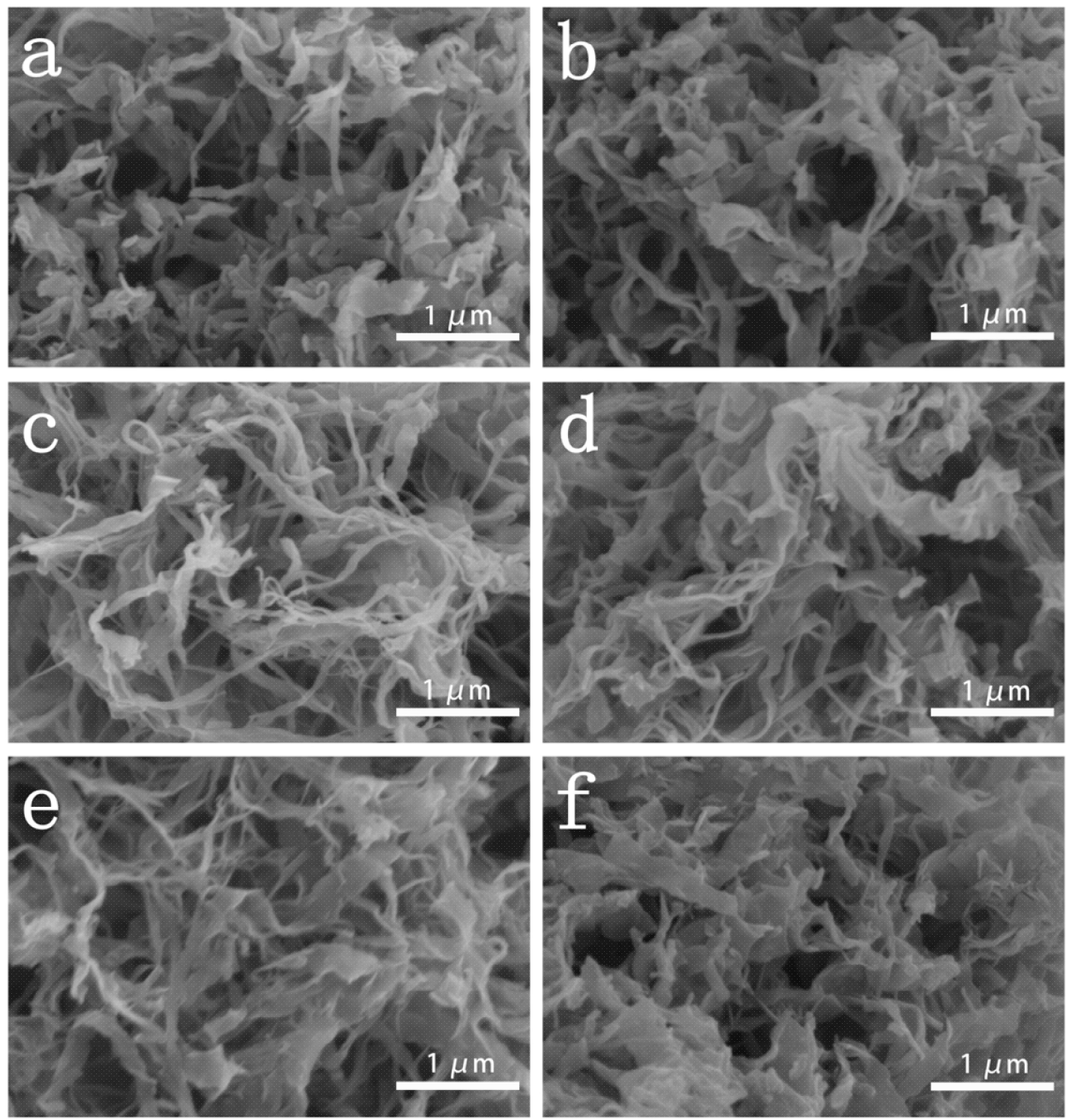

Fig. 5.FESEM images of (a) CsCNBs-800/S, (b)CsCNBs-800*3/S, (c) CsCNBs- 800*4/S, (d) CsCNBs-800*5/S, (e) CsCNBs-950-40, (f) CsCNBs-800*4/S90. 

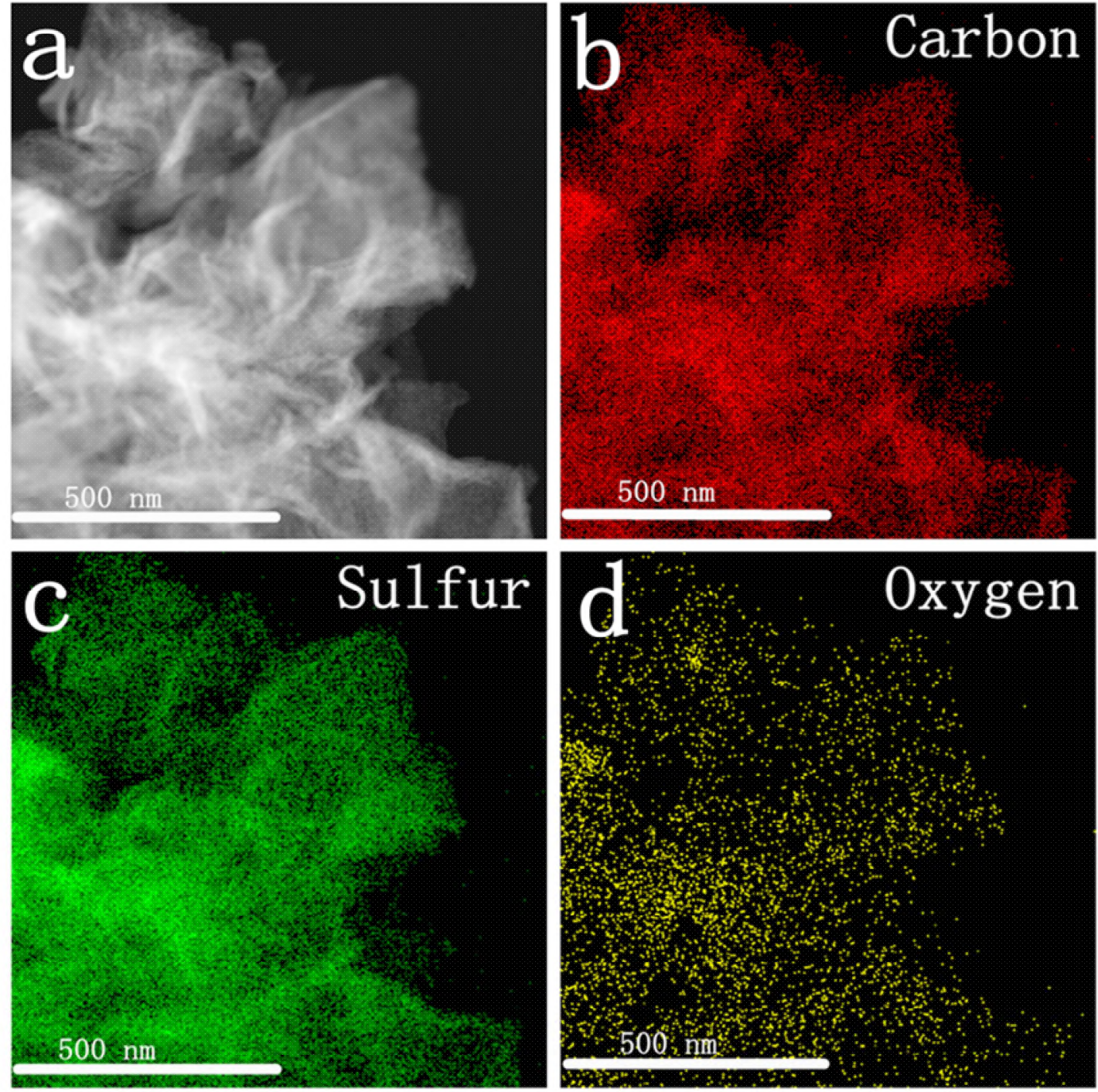

Fig. 6. The EDX images of CsCNBs- $800 * 4 / \mathrm{S}$ composite.
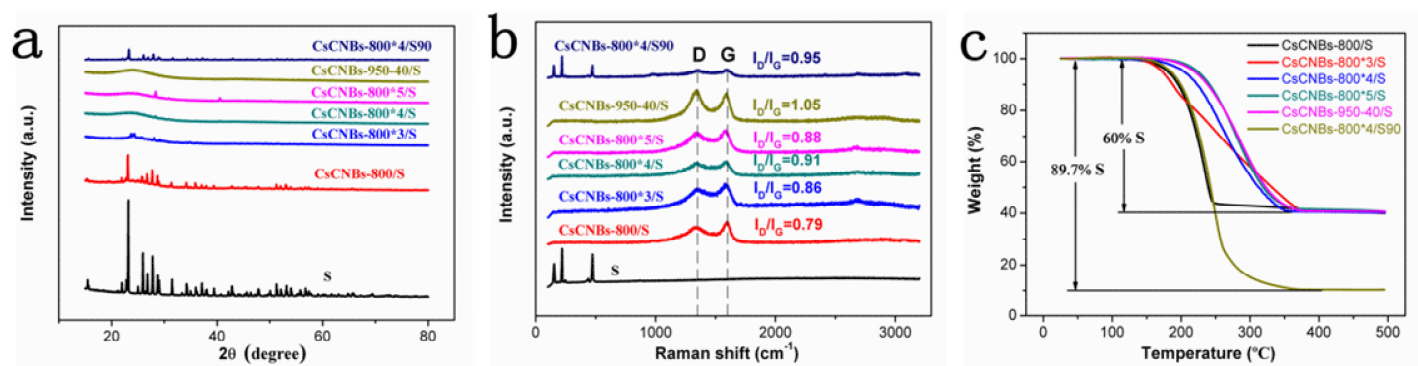

Fig. 7. (a) XRD patterns, (b) Raman spectra and (c) TGA of all carbon-sulfur composites.

The XRD patterns of pure sulfur and all carbon/sulfur composites are shown in Fig.7a. Obvious sulfur characteristic peaks can be seen in the XRD curves of CsCNBs-800/S and CsCNBs-800*3/S, which masks some characteristic peaks of carbon materials. On the XRD curves of CsCNBs-800*4/S and CsCNBs-950-40/S, the sulfur characteristic peaks disappear because the sulfur, dispersed in the micropores and mesopores, is inhibited by the pores and cannot be crystallized. However, there are a few sulfur characteristic peaks in the XRD curve of 
CsCNBs-800*5/S, because the pore volume of CsCNBs-800*5 is not large enough to completely accommodate and adsorb sulfur. The sulfur characteristic peak in the XRD curve of the CsCNBs-800*4/S90 composite completely obscures the carbon characteristic peak because the pore structure in the CsCNBs-800*4 material cannot completely contain sulfur and the sulfur is distributed on the surface of the nanobelts. The Raman spectra of pure sulfur and all carbon/sulfur composites are shown in Fig. 7b. All CsCNBs/S composites with $60 \%$ sulfur content showed only $\mathrm{D}$ band and $\mathrm{G}$ band of amorphous carbon, and no sulfur characteristic peak was observed. However, a strong sulfur characteristic peak can be seen in the Raman spectrum of the CsCNBs-800*4/S90 composite due to its high sulfur content. Compared with the intensity ratio of the corresponding CsCNBs, the $\mathrm{R}$ values of all $\mathrm{CsCNBs} / \mathrm{S}$ composites are slightly reduced, indicating that sulfur is present in the pore structure of all samples, thereby increasing the degree of order. Fig. $7 \mathrm{c}$ shows the TGA of all carbon/sulfur composites. The actual sulfur content of CsCNBs-800*4/S90 composite is $89.7 \%$, and other composites are close to $60 \%$. The sulfur contents of all composites have reached the expected setting. The weight loss curves of CsCNBs-800/S, CsCNBs-800*3/S and CsCNBs-800*4/S90 composites are divided into two stages. The weight loss steps in the range of 150 250 ${ }^{\circ} \mathrm{C}$ and $250 \sim 400{ }^{\circ} \mathrm{C}$ are attributed to the volatilization of the sulfur outside and inside micropores/mesopores, respectively. Since the pore volume of CsCNBs-800 is small $\left(0.31 \mathrm{~cm}^{3} \mathrm{~g}^{-1}\right)$, the sulfur inside the micropores/mesopores is less than $5 \%$ (based on the whole composite). Due to the smaller pore volume of CsCNBs-800*3 $\left(0.60 \mathrm{~cm}^{3} \mathrm{~g}^{-1}\right)$, the sulfur inside the micropores/mesopores is around $40 \%$ (based on the whole composite). During the temperature rises from 150 to $250{ }^{\circ} \mathrm{C}$, about $70 \%$ (based on the entire complex) of sulfur is volatilized in the CsCNBs-800*4/S90 composite, which indicates that the high pore volume of the CsCNBs- $800 * 4$ is not sufficient to accommodate ultrahigh content of sulfur. However, the weight loss curves of CsCNBs-800*4/S, CsCNBs-800*5/S and CsCNBs-950-40/S composites have only one weight loss 
process, because the pore volume of CsCNBs-800*4, CsCNBs-800*5 and CsCNBs-950-40 is large enough to encapsulate most of the active substance sulfur. Table S3 shows the elemental analysis data of each CsCNBs/S composite, which is basically consistent with the TGA results.

To evaluate the electrochemical properties of these samples, all CsCNBs/S composites are assembled into a button-type LiSBs, in which a lithium metal sheet is used as a negative electrode. The assembling batteries are cycled at various current rates in a voltage window of $1.8-2.8 \mathrm{~V}$ [48-50]. Figure 8a shows the initial discharge/charge curves of each battery at $0.1 \mathrm{C}$ [51]. The discharge curves of all samples display two platforms of discharge at $2.3 \mathrm{~V}$ and $2.1 \mathrm{~V}$, corresponding to the characteristic discharge platforms of sulfur [52-53]. Comparing with other composites, both discharge platforms of CsCNBs-800*4/S90 are moving down because the ultrahigh content of sulfur reduces the conductivity of the entire composite. There is a small platform in the discharge curve of CsCNBs-800*X $(\mathrm{X}=3,4,5) / \mathrm{S}$, and CsCNBs-950-40/S at approximately $1.8 \mathrm{~V}$, which is attributed to the consumption of $\mathrm{LiNO}_{3}$ in the electrolyte [54-55]. The reduction of $\mathrm{LiNO}_{3}$ may be catalyzed by the bare carbon surface with a high specific surface area at a small current rate. Fig.8b shows the cycle curve of each battery at $0.1 \mathrm{C}$. The initial discharge specific capacities of CsCNBs-800/S, CsCNBs-800*X(X=3, 4, 5)/S, CsCNBs-950-40/S and CsCNBs-800*4/S90 are 839, 1064, 1245, 1170, 1371 and $785 \mathrm{~mA} \mathrm{~h} \mathrm{~g}^{-1}$, respectively. It can be found that the larger the specific surface area and pore volume of the CsCNBs material, the higher the initial discharge specific capacity of the corresponding $\mathrm{CsCNBs} / \mathrm{S}$ composite. For the CsCNBs-800*4/S90 composite, the sulfur content is $90 \%$, which causes the conductivity of the positive electrode to decrease, resulting in most of the available sulfur not being fully utilized. Therefore, a lower but acceptable discharge specific capacity is exhibited. After 200 cycles of charge/discharge test, the capacity retention rates of CsCNBs-800/S, CsCNBs-800*X(X=3, 4, 5)/S, 
respectively. The CsCNBs-800*4/S exhibits the best long-term cycling stability at $0.1 \mathrm{C}$ with a capacity decay rate as low as $0.162 \%$ per cycle. When the sulfur content is as high as $90 \%$ (CsCNBs-800*4/S90), an excellent long-term cycling stability is still retained. However, the capacity decay rate of CsCNBs-950-40/S composite with almost specific surface area and pore volume is $0.280 \%$ per cycle. In short, the superior cycle stability of CsCNBs-800*4/S composite can be attributed to its rich polar oxygen-containing functional groups, which have a stronger adsorption effect on polysulfides and increase the utilization of sulfur [56-57].
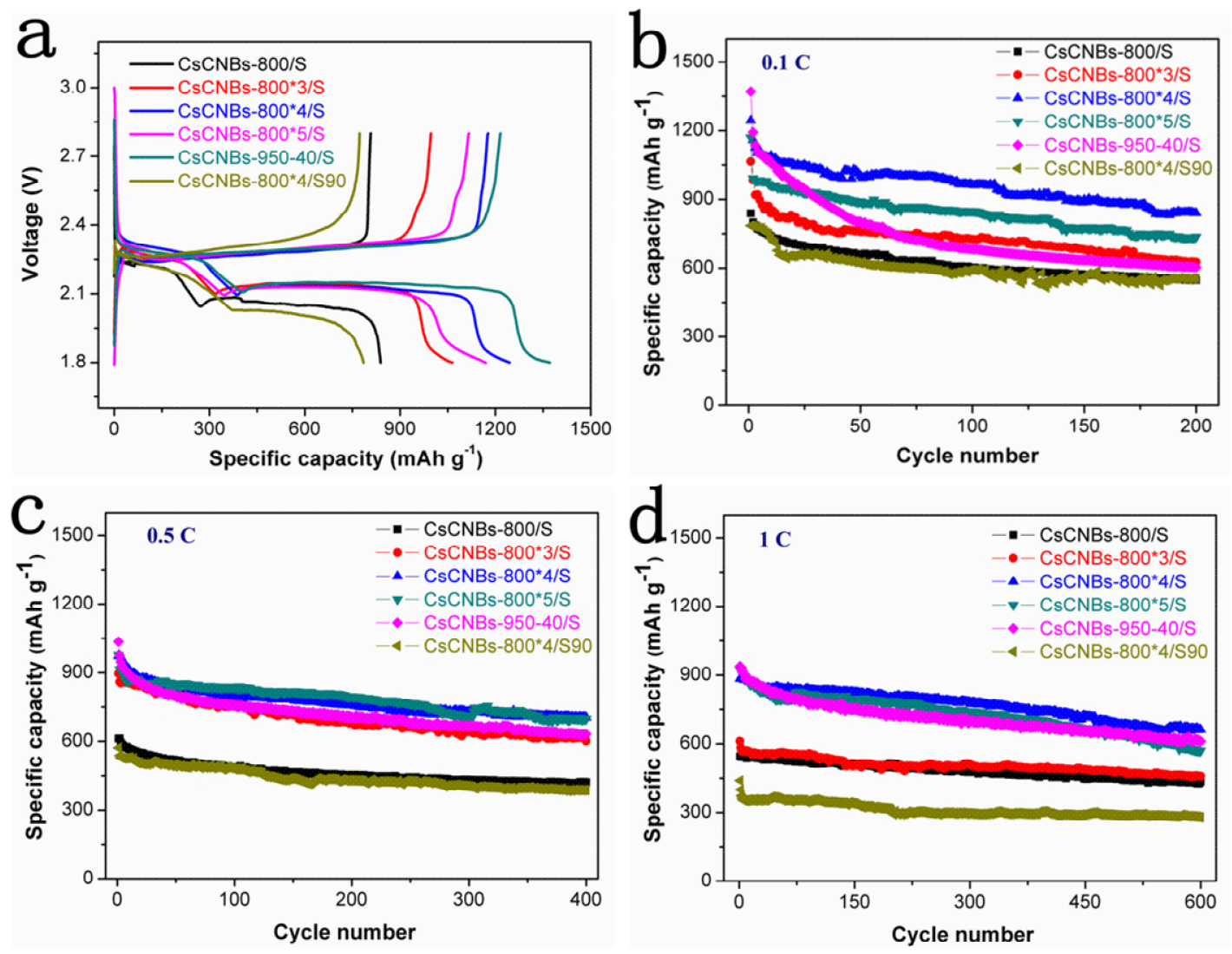

Fig. 8. Electrochemical performance of all composites: (a) the initial discharge/charge curves and the cycle performances at (b) $0.1 \mathrm{C}$, (c) $0.5 \mathrm{C}$ and (d) $1 \mathrm{C}$.

As shown in Fig. 8c, the initial discharge specific capacities of CsCNBs-800/S, CsCNBs-800*X(X=3, 4, 5)/S, CsCNBs-950-40/S and CsCNBs-800*4/S90 are 611, 895, 974, 977, 1035 and $572 \mathrm{~mA} \mathrm{~h} \mathrm{~g}^{-1}$, respectively. After 400 cycles, the capacity retention rates are $69.0 \%, 67.2 \%$, $72.4 \%, 71.5 \%, 61.0 \%$ and $67.7 \%$, respectively. Each battery exhibits an excellent long-term cycling stability, especially for the battery made from the CsCNBs-800*4/S. The cyclic curve of each battery test at $1 \mathrm{C}$ current rate is shown in Fig. 8d. The initial discharge specific capacities of 
CsCNBs-800/S, CsCNBs-800*X(X=3, 4, 5)/S, CsCNBs-950-40/S and CsCNBs-800*4/S90 are 544,

$611,884,922,939$ and $440 \mathrm{~mA} \mathrm{~h} \mathrm{~g}^{-1}$, respectively. After 600 cycles, the capacity retention rates are $80.0 \%, 75.1 \%, 75.0 \%, 61.8 \%, 65.0 \%$ and $63.4 \%$, respectively. Discharged at high currents, CsCNBs-800*4/S composites still exhibit high capacity and excellent long-term cycle stability. Even with a sulfur content of $90 \%$, the CsCNBs-800*4/S90 electrode obtains acceptable electrochemical performance.
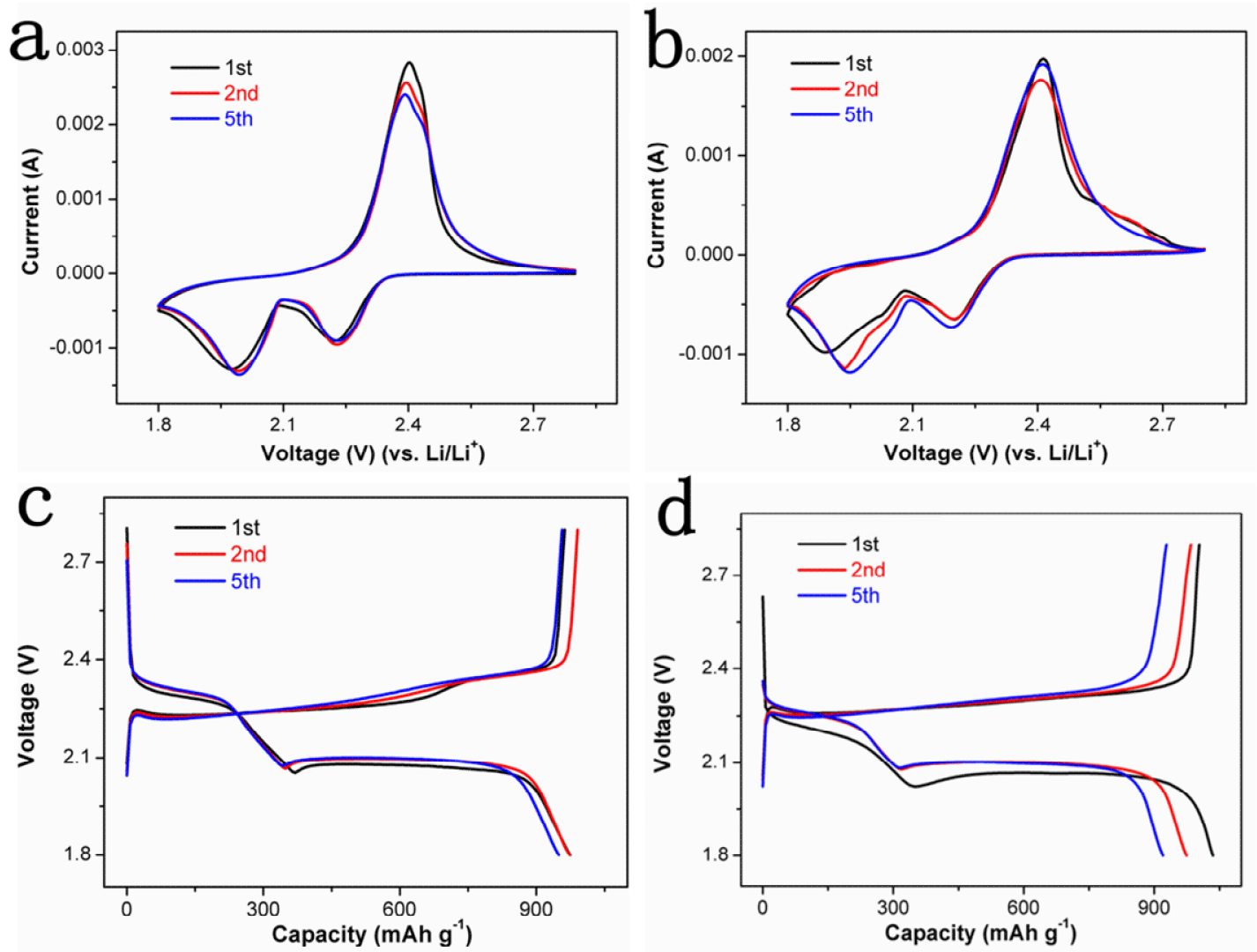

Fig. 9. The CV curves of (a) CsCNBs-800*4/S and (b) CsCNBs-950-40/S measured between 1.8 and $2.8 \mathrm{~V}$ versus $\mathrm{Li}^{+} / \mathrm{Li}$ at a sweep rate of $0.2 \mathrm{mV} \mathrm{s}^{-1}$. Charge/discharge curves of (c) CsCNBs-800*4/S and (d) CsCNBs-950-40/S at $0.5 \mathrm{C}$.

Table S4 is the electrochemical performance of the CsCNBs-800*4/S and CsCNBs-950-40/S in LiSBs. It is found that the CsCNBs-800*4/S composite exhibits a low initial discharge specific capacity, but the decay rate of per cycle capacity is much lower than that of CsCNBs-950-40/S composites, especially at low current cycles. Porous carbon materials, CsCNBs-800*4 and CsCNBs-950-40, have similar specific surface area and pore volume, three-dimensional conductive network, pore structure, etc. The biggest difference is the difference in oxygen content. As can be 
seen from Fig. 4f, CsCNBs-800*4 sample has rich polar oxygen-containing functional groups (C-OH/C-O-C, hydroxyl/epoxide).These functional groups are favorable for adsorbing lithium polysulfide during the cycles of a lithium-sulfur battery, suppressing the shuttle effect, and improving the utilization rate of the active material sulfur. Adequate evidence for the excellent electrochemical performance is exhibited by CsCNBs- $800 * 4$ sample.

As mentioned above, the CsCNBs- $800 * 4 / \mathrm{S}$ composite exhibits the best electrochemical performance. Therefore, the electrochemical performances of CsCNBs-800*4/S and CsCNBs-950-40/S composites are further investigated by CV testing [58-59]. As shown in Fig. 9a, CsCNBs-800*4/S composite displays two peaks at 2.25 and $1.95 \mathrm{~V}$ during the first voltage scan. During the second voltage sweep, the peak at $2.25 \mathrm{~V}$ is unchanged, while the peak at $1.95 \mathrm{~V}$ is moved to $2.00 \mathrm{~V}$. During the fifth voltage sweep, the curves are substantially coincident and the peaks are unchanged, indicating that good electrochemical stability and reversibility. Fig. 9c shows the first, second, and fifth discharge/charge curves at $0.5 \mathrm{C}$, which show that the migration of the discharge platform is consistent with the CV curve and the capacity attenuation is small. Fig. $9 \mathrm{~b}$ shows the CV curves of CsCNBs-800*4/S composite. During the first voltage scan, there are two peaks at 2.20 and $1.90 \mathrm{~V}$. During the second and fifth voltage sweeps, the peak of high voltage discharge platform is essentially unchanged, and the peak of low voltage discharge platform migrates to 1.95 V. Fig. 9 d shows the first, second, and fifth discharge/charge curves at $0.5 \mathrm{C}$, which show that the displacement of the discharge platform is consistent with the CV curve, and the capacity decays faster. The CV curves further indicate that CsCNBs-800*4/S composite has a small capacity attenuation and high cycle stability. 

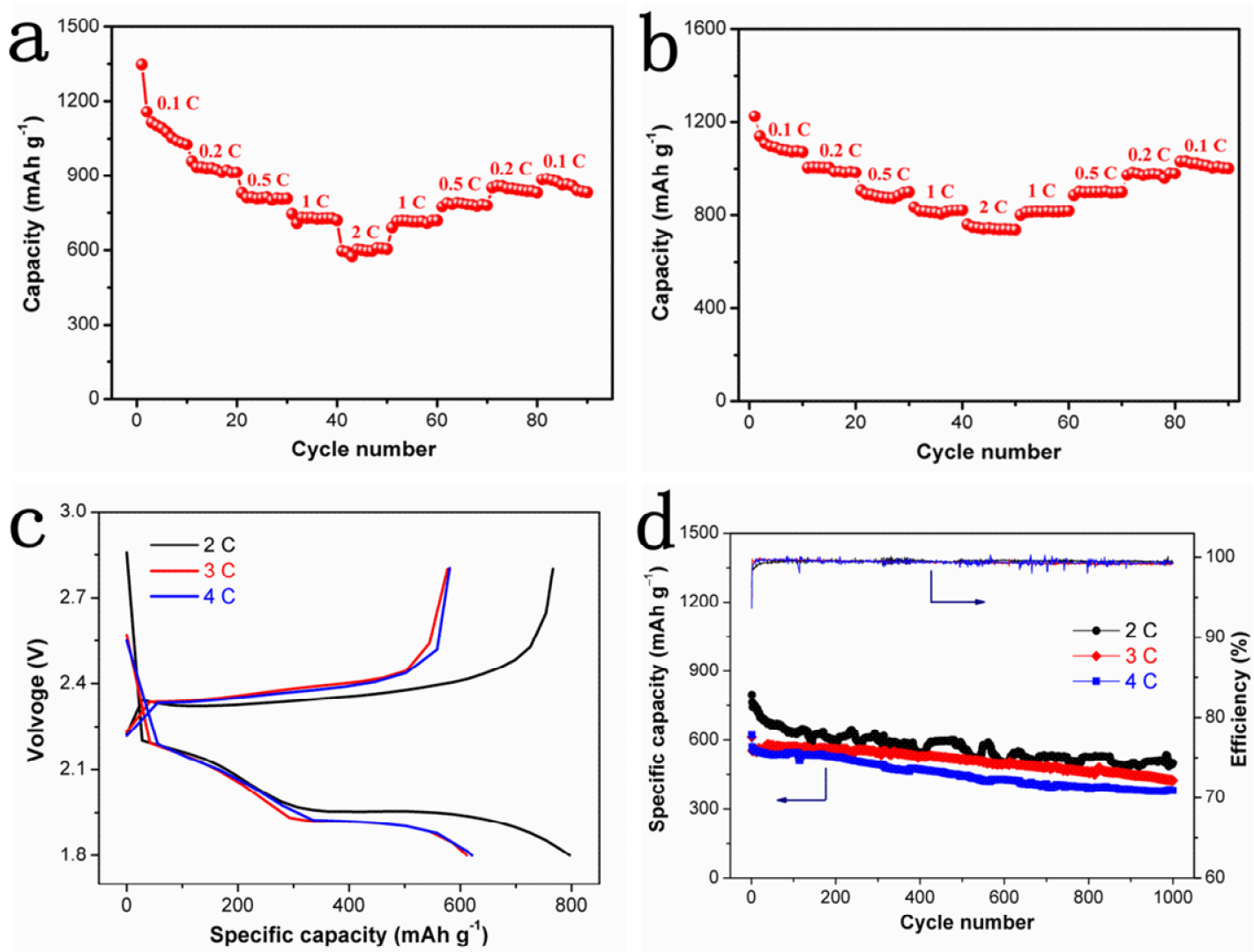

Fig. 10. The rate-cycle curves of (a) CsCNBs-950-40/S and (b) CsCNBs-800*4/S composite. (c) The initial discharge/charge curves and (d) the cycle performances and coulombic efficiencies of CsCNBs-800*4/S composite at 2 C, 3 C and 4 C.

In order to visually characterize the rate cycling characteristics, the batteries of CsCNBs-950-40/S and CsCNBs-800*4/S composites are tested for charge/discharge cycles at various current rates [60]. Fig. 10a shows the rate performance of CsCNBs-950-40/S composite. At $0.1 \mathrm{C}$, the discharge specific capacity of CsCNBs-950-40/S rapidly decreased from $1348 \mathrm{~mA} \mathrm{~h} \mathrm{~g}^{-1}$ to $1158 \mathrm{~mA} \mathrm{~h} \mathrm{~g}^{-1}$. The capacities of CsCNBs-950-40/S at $0.2,0.5,1$, and $2 \mathrm{C}$ are 958, 832, 746, and $597 \mathrm{~mA} \mathrm{~h} \mathrm{~g}^{-1}$, respectively. When the current rate isswitched back to 1 and $0.5 \mathrm{C}$, the capacities can be largely recovered. However, when the current rate is switched back to 0.2 and $0.1 \mathrm{C}$, the capacity loss is large. The rate performance of CsCNBs-800*4/S composite is shown in Fig. 10b. At $0.1 \mathrm{C}$, the discharge specific capacity of CsCNBs-950-40/S rapidly decreased from $1225 \mathrm{~mA} \mathrm{~h} \mathrm{~g}^{-1}$ to 1141 $\mathrm{mA} \mathrm{h} \mathrm{g}^{-1}$. The capacities of CsCNBs-800*4/S at $0.2,0.5,1$, and $2 \mathrm{C}$ are about 1005, 907, 835, and $761 \mathrm{~mA} \mathrm{~h} \mathrm{~g}^{-1}$, respectively. When the current rate is switched back to $1 \mathrm{C}, 0.5 \mathrm{C}, 0.2 \mathrm{C}$, and $0.1 \mathrm{C}$, the discharge specific capacity is greatly restored. At $0.1 \mathrm{C}$, discharge specific capacity is $1031 \mathrm{~mA}$ 
$\mathrm{h} \mathrm{g}^{-1}$, indicating that the CsCNBs-800*4/S composite has an excellent rate cycling performance.

In order to characterize the high current discharge performance, the batteries of CsCNBs-800*4/S are tested at $2 \mathrm{C}, 3 \mathrm{C}$ and $4 \mathrm{C}[11,61,62]$. The initial discharge/charge curve is shown in Fig. 10c. All initial discharge curves show two sulfur discharge platforms at $2.20 \mathrm{~V}$ and $1.95 \mathrm{~V}$. At $2 \mathrm{C}, 3 \mathrm{C}$ and $4 \mathrm{C}$, the initial discharge ratio capacities are 797, 612 and $621 \mathrm{~mA} \mathrm{~h} \mathrm{~g}^{-1}$, respectively. The coulombic efficiency decreased in turn $96.2 \%, 94.5 \%$ and $93.6 \%$, respectively. The cycle performances and coulombic efficiencies of CsCNBs-800*4/S batteries at $2 \mathrm{C}, 3 \mathrm{C}$ and 4 $\mathrm{C}$ are shown in Fig.10d. After 1000 cycles of charge and discharge, the discharge specific capacity of the battery at each current rate was reduced to 500,425 and $381 \mathrm{~mA} \mathrm{~h} \mathrm{~g}^{-1}$, respectively. The capacity decay rate was as low as $0.037 \%, 0.030 \%$ and $0.039 \%$ per cycle, indicating a slow loss of sulfur during the cycle. Although the first coulombic efficiency of CsCNBs-800*4/S composites at $2 \mathrm{C}, 3 \mathrm{C}$ and $4 \mathrm{C}$ is lower, they are very close to $100 \%$ from the second to the $1000^{\text {th }}$ cycle, indicating that the shuttle effect of sulfides is effectively suppressed during cycles.

Compared with CsCNBs-950-40 material, CsCNBs-800*4 material exhibits superior electrochemical performance in LiSBs, including high capacity, excellent rate performance and long-term high cycling stability, which can be ascribed to four main factors. First, the highly conductive three-dimensional network structure of the nanobelts promotes the rapid transmission of electrons and improves the overall conductivity of the positive electrode. The EIS results of CsCNBs-950-40 and CsCNBs-800*4 have further investigated this point (supplementary materials Fig.6). Second, the nanobelts are very thin (the range of 10-30 nm), and the diffusion path of lithium ions can be reduced to half the thickness of the nanobelts (about $10 \mathrm{~nm}$ ), resulting in rapid lithium ion kinetics. Third, the synergistic effect of multi-stage pores stores sulfur, has physical adsorption to lithium polysulfides, and inhibits the occurrence of shuttle effect. The foregoing three mechanism factors of CsCNBs-800*4 material are basically similar to those of CsCNBs-950-40. 
Fourth, the polar oxygen-containing (hydroxyl/epoxide) functional groups can strongly bind to lithium polysulfides and have a strong chemisorption to polysulfides, which can prevent polysulfides from dissolving into the electrolyte and inhibit the generation of shuttle effect. This is the core of CsCNBs-800*4 material with superior rate performance and cycle stability. In addition, the electrochemical performance of other related carbon/sulfur composite were exhibited in Supplementary materials Table S5, and these results further prove the outstanding electrochemical performance of our prepared carbon nanobelts in LiSBs.

\section{Conclusion}

In summary, CsCNBs-800*4 with ultrahigh SSA $\left(2300 \mathrm{~m}^{2} \mathrm{~g}^{-1}\right)$ and large pore volume (up to $1.29 \mathrm{~cm}^{3} \mathrm{~g}^{-1}$ ) has been successfully fabricated from phenolic resin based nanobelts by carbonization and $\mathrm{KOH}$ activation. The product possesses hierarchically porous structure, three-dimensional conductive network framework, and polar oxygen-rich groups, which are very befitting to load sulfur for excellent cycle-stability of LiSBs. As a result, CsCNBs-800*4/S cells display an ultrahigh initial discharge capacity of $1245 \mathrm{~mA} \mathrm{~h} \mathrm{~g}^{-1}$ and ultralow capacity decay rate as low as $0.162 \%$ per cycle after 200 cycles at $0.1 \mathrm{C}$. Even at high current rate of $4 \mathrm{C}$, the cells still exhibit a high initial discharge capacity $\left(621 \mathrm{~mA} \mathrm{~h} \mathrm{~g}^{-1}\right)$ and ultralow capacity decay rate (only $0.039 \%$ per cycle) after 1000 cycles. These encouraging results indicate that polar oxygen-containing functional groups are important for improving the electrochemical performance of carbons. The oxygen-doped carbon nanobelts have an excellent energy storage potential in the field of energy storage.

\section{Acknowledgements}

This work was financially supported by the National Natural Science Foundation of China (Nos.51202150, 51272161 and 21703141), the Science and Technology R\&D Program of Shenzhen the Program of Introducing Innovative Research Team in Dongguan (No.2014607109).

\section{References}


[1] P.G. Bruce, B. Scrosati, and J.M. Tarascon, Nanomaterials for rechargeable lithium batteries, Angew Chem Int Ed Engl, 47, 2930 (2008).

[2] B. Scrosati and J. Garche, Lithium batteries: Status, prospects and future, Journal of

Power Sources, 195, 2419 (2010).

[3] Q. Pang, D. Kundu, and L. F. Nazar, A graphene-like metallic cathode host for longlife and high-loading lithium-sulfur batteries, Materials Horizons, 3, 130 (2016).

[4] Q. Pang, J. Tang, H. Huang, X. Liang, C. Hart, K. C. Tam, and L. F. Nazar, A nitrogen 548 and sulfur dual-doped carbon derived from polyrhodanine@cellulose for advanced lithium-sulfur batteries, Adv Mater, 27, 6021 (2015).

[5] B. Papandrea, X. Xu, Y. Xu, C.-Y. Chen, Z. Lin, G. Wang, Y. Luo, M. Liu, Y. Huang, 551 L. Mai, and X. Duan, Three-dimensional graphene framework with ultra-high sulfur content for a robust lithium-sulfur battery, Nano Research, 9, 240(2016).

[6] X. Ji, L. F. Nazar, Advances in Li-S batteries, Journal of Materials Chemistry, 20 (2010).

[7] X. Tao, J. Wang, C. Liu, H. Wang, H. Yao, G. Zheng, Z. W. Seh, Q. Cai, W. Li, G. Zhou, C. Zu, Y. Cui, Balancing surface adsorption and diffusion of lithium-polysulfides on nonconductive oxides for lithium-sulfur battery design, Nat Commun, 7 (2016) 11203.

[8] Z. Wei Seh, W. Li, J. J. Cha, G. Zheng, Y. Yang, M. T. McDowell, P. C. Hsu, Y. Cui, Sulphur-TiO ${ }_{2}$ yolk-shell nanoarchitecture with internal void space for long-cycle lithium-sulphur batteries, Nat Commun, 4 (2013) 1331.

[9] P. Adelhelm, P. Hartmann, C. L. Bender, M. Busche, C. Eufinger, J. Janek, From lithium to sodium: cell chemistry of room temperature sodium-air and sodium-sulfur batteries, Beilstein J Nanotechnol, 6 (2015) 1016-1055.

[11] P. G. Bruce, S. A. Freunberger, L. J. Hardwick, J. M. Tarascon, Li-O $\mathrm{O}_{2}$ and Li-S batteries with high energy storage, Nat Mater, 11 (2011) 19-29.

[12] S. Evers, L. F. Nazar, Graphene-enveloped sulfur in a one pot reaction: a cathode with good coulombic efficiency and high practical sulfur content, Chem Commun (Camb), 48 (2012) 1233-1235.

[13] S. Evers, L. F. Nazar, New approaches for high energy density lithium-sulfur battery cathodes, Acc Chem Res, 46 (2013) 1135-1143.

[14] L. Ma, H. L. Zhuang, S. Wei, K. E. Hendrickson, M. S. Kim, G. Cohn, R. G. Hennig, L. A. Archer, Enhanced Li-S Batteries Using Amine-Functionalized Carbon Nanotubes in the Cathode, ACS Nano, 10 (2016) 1050-1059.

[16] F. Wu, J. Qian, R. Chen, J. Lu, L. Li, H. Wu, J. Chen, T. Zhao, Y. Ye, K. Amine, An effective approach to protect lithium anode and improve cycle performance for Li-S batteries, ACS Appl Mater Interfaces, 6 (2014) 15542-15549.

[17] R. Xu, I. Belharouak, X. Zhang, R. Chamoun, C. Yu, Y. Ren, A. Nie, R. Shahbazian-Yassar, J. Lu, J. C. Li, K. Amine, Insight into sulfur reactions in Li-S batteries, ACS Appl Mater Interfaces, 6 (2014) 21938-21945.

[18] J. Wang, S. Kaskel, KOH activation of carbon-based materials for energy storage, Journal of Materials Chemistry, 22 (2012).

[19] C. Hoffmann, S. Thieme, J. Bruckner, M. Oschatz, T. Biemelt, G. Mondin, H. Althues, S. Kaskel, Nanocasting hierarchical carbide-derived carbons in nanostructured opal assemblies for high-performance cathodes in lithium-sulfur batteries, ACS Nano, 8 (2014) 12130-12140.

[20] J. Hou, C. Cao, F. Idrees, X. Ma, Hierarchical porous nitrogen-doped carbon nanosheets derived from silk for ultrahigh-capacity battery anodes and supercapacitors, ACS Nano, 9 (2015) 2556-2564.

[21] P. Strubel, S. Thieme, T. Biemelt, A. Helmer, M. Oschatz, J. Brückner, H. Althues, S. Kaskel, $\mathrm{ZnO}$ Hard Templating for Synthesis of Hierarchical Porous Carbons with Tailored Porosity and 
High Performance in Lithium-Sulfur Battery, Advanced Functional Materials, 25 (2015) 287-297.

[22] S. Z. Zeng, Y. Yao, L. Huang, H. Wu, B. Peng, Q. Zhang, X. Li, L. Yu, S. Liu, W. Tu, T. Lan, X. Zeng, J. Zou, Facile Synthesis of Ultrahigh-Surface-Area Hollow Carbon Nanospheres and their Application in Lithium-Sulfur Batteries, Chemistry, 24 (2018) 1988-1997.

[23] Z. W. Seh, H. Wang, N. Liu, G. Zheng, W. Li, H. Yao, Y. Cui, High-capacity Li2S-graphene oxide composite cathodes with stable cycling performance, Chemical Science, 5 (2014).

[24] G. Zhou, L. C. Yin, D. W. Wang, L. Li, S. Pei, I. R. Gentle, F. Li, H. M. Cheng, Fibrous hybrid of graphene and sulfur nanocrystals for high-performance lithium-sulfur batteries, ACS Nano, 7 (2013) 5367-5375.

[25] F. Chen, J. Yang, T. Bai, B. Long, X. Zhou, Biomass waste-derived honeycomb-like nitrogen and oxygen dual-doped porous carbon for high performance lithium-sulfur batteries, Electrochimica Acta, 192 (2016) 99-109.

[26] G. He, X. Ji, L. Nazar, High “C” rate Li-S cathodes: sulfur imbibed bimodal porous carbons, Energy \& Environmental Science, 4 (2011).

[27] N. Jayaprakash, J. Shen, S. S. Moganty, A. Corona, L. A. Archer, Porous hollow carbon@sulfur composites for high-power lithium-sulfur batteries, Angew Chem Int Ed Engl, 50 (2011) 5904-5908.

[28] J.T. Lee, Y. Zhao, S. Thieme, H. Kim, M. Oschatz, L. Borchardt, A. Magasinski, W. I. Cho, S. Kaskel, G. Yushin, Sulfur-infiltrated micro- and mesoporous silicon carbide-derived carbon cathode for high-performance lithium sulfur batteries, Adv Mater, 25 (2013) 4573-4579.

[29] M. Oschatz, S. Thieme, L. Borchardt, M. R. Lohe, T. Biemelt, J. Bruckner, H. Althues, S. Kaskel, A new route for the preparation of mesoporous carbon materials with high performance in lithium-sulphur battery cathodes, Chem Commun (Camb), 49 (2013) 5832-5834.

[30] J. Schuster, G. He, B. Mandlmeier, T. Yim, K. T. Lee, T. Bein, L. F. Nazar, Spherical ordered mesoporous carbon nanoparticles with high porosity for lithium-sulfur batteries, Angew Chem Int Ed Engl, 51 (2012) 3591-3595.

[31] D. W. Wang, G. Zhou, F. Li, K. H. Wu, G. Q. Lu, H. M. Cheng, I. R. Gentle, A microporous-mesoporous carbon with graphitic structure for a high-rate stable sulfur cathode in carbonate solvent-based Li-S batteries, Phys Chem Chem Phys, 14 (2012) 8703-8710.

[32] D. L. Vu, J. S. Seo, H. Y. Lee, J. W. Lee, Activated carbon with hierarchical micro-mesoporous structure obtained from rice husk and its application for lithium-sulfur batteries, RSC Advances, 7 (2017) 4144-4151.

[32] B. Zhang, X. Qin, G.R. Li, X. P. Gao, Enhancement of long stability of sulfur cathode by encapsulating sulfur into micropores of carbon spheres, Energy \& Environmental Science, 3 (2010).

[33] S. Zhang, M. Zheng, Z. Lin, N. Li, Y. Liu, B. Zhao, H. Pang, J. Cao, P. He, Y. Shi, Activated carbon with ultrahigh specific surface area synthesized from natural plant material for lithium-sulfur batteries, J. Mater. Chem. A, 2 (2014) 15889-15896.

[34] S. Zhang, M. Zheng, Z. Lin, N. Li, Y. Liu, B. Zhao, H. Pang, J. Cao, P. He, and Y. Shi, Activated carbon with ultrahigh specific surface area synthesized from natural plant material for lithium-sulfur batteries, J. Mater. Chem. A, 2, 15889 (2014).

[35] R. Elazari, G. Salitra, A. Garsuch, A. Panchenko, D. Aurbach, Sulfur-impregnated activated carbon fiber cloth as a binder-free cathode for rechargeable Li-S batteries, Adv Mater, 23 (2011) 5641-5644.

[36] M. Raja, N. Angulakshmi, A. M. Stephan, Sisal-derived activated carbons for cost-effective lithium-sulfur batteries, RSC Advances, 6 (2016) 13772-13779. 
[37] A. Bhargav, M. E. Bell, J. Karty, Y. Cui, and Y. Fu, A Class of Organopolysulfides

As Liquid Cathode Materials for High-Energy-Density Lithium Batteries, ACS Appl Mater Interfaces, 10,21084 (2018).

[38] Y. Liu, J. S. Xue, T. Zheng, and J. R. Dahn, Mechanism of lithium insertion in hard carbons prepared by pyrolysis of epoxy resins, Carbon, 4, 193 (1996).

[40] S. Gupta, B. R. Weiner, G. Morell, Investigations of the electron field emission properties and microstructure correlation in sulfur-incorporated nanocrystalline carbon thin films, Journal of Applied Physics, 91 (2002).

[41] L. F. Chen, Z. H. Huang, H. W. Liang, W. T. Yao, Z. Y. Yu, S. H. Yu, Flexible all-solid-state high-power supercapacitor fabricated with nitrogen-doped carbon nanofiber electrode material derived from bacterial cellulose, Energy \& Environmental Science, 6 (2013).

[42] C. H. Choi, S. H. Park, S. I. Woo, Binary and ternary doping of nitrogen, boron, and phosphorus into carbon for enhancing electrochemical oxygen reduction activity, ACS Nano, 6 (2012) 7084-7091.

[43] D. H. Seo, A. E. Rider, Z. J. Han, S. Kumar, K. K. Ostrikov, Plasma break-down and re-build: same functional vertical graphenes from diverse natural precursors, Adv Mater, 25 (2013) 5638-5642.

[44] A. C. Ferrari, Raman spectroscopy of graphene and graphite: Disorder, electron- 666 phonon coupling, doping and nonadiabatic effects, Solid State Communications, 143, 47 (2007). 668

[45] I. Pocsik, M. Hundhausen, M. Koos, and L. Ley, Origin of the D peak in the Raman spectrum of microcrystalline graphite, Journal of Non-Crystalline Solids, 227-230, 1083 (1998).

[46] M. H. Rummeli, A. Bachmatiuk, A. Scott, F. Borrnert, J. H. Warner, V. Hoffman, J.-H. Lin, G. Cuniberti, and B. Buchner, Direct low-temperature nanographene CVD synthesis over a dielectric insulator, ACS Nano, 4, 4206 (2010).674

[47] R. P. Vidano, D. B. Fischbach, L. J. Willis, and T. M. Loehr, Observation of Raman band shiting with excitation wavelength for carbons and graphites Solid State Comwnications, 39, 341 (1981).

[48] J. Hassoun, F. Croce, M. Armand, B. Scrosati, Investigation of the $\mathrm{O}_{2}$ Electrochemistry in a Polymer Electrolyte Solid-State Cell, Angewandte Chemie, 123 (2011) 3055-3058.

[49] K. Oyaizu, A. Hatemata, W. Choi, H. Nishide, Redox-active polyimide/carbon nanocomposite electrodes for reversible charge storage at negative potentials: expanding the functional horizon of polyimides, Journal of Materials Chemistry, 20 (2010).

[50] Q. Zhao, Z. Zhu, J. Chen, Molecular Engineering with Organic Carbonyl Electrode Materials for Advanced Stationary and Redox Flow Rechargeable Batteries, Adv Mater, 29 (2017).

[51] J. H. Park, K. M. Choi, D. K. Lee, B. C. Moon, S. R. Shin, M. K. Song, J. K. Kang, Encapsulation of redox polysulphides via chemical interaction with nitrogen atoms in the organic linkers of metal-organic framework nanocrystals, Sci Rep, 6 (2016) 25555.

[52] G. Hu, Z. Sun, C. Shi, R. Fang, J. Chen, P. Hou, C. Liu, H. M. Cheng, F. Li, A Sulfur-Rich Copolymer@CNT Hybrid Cathode with Dual-Confinement of Polysulfides for High-Performance Lithium-Sulfur Batteries, Adv Mater, 29 (2017).

[53] J. Q. Huang, Q. Zhang, H. J. Peng, X. Y. Liu, W. Z. Qian, F. Wei, Ionic shield for polysulfides towards highly-stable lithium-sulfur batteries, Energy Environ. Sci., 7 (2014) 347-353.

[54] A. Rosenman, E. Markevich, G. Salitra, D. Aurbach, A. Garsuch, F. F. Chesneau, Review on Li-Sulfur Battery Systems: an Integral Perspective, Advanced Energy Materials, 5 (2015).

[55] S. S. Zhang, Role of $\mathrm{LiNO}_{3}$ in rechargeable lithium/sulfur battery, Electrochimica Acta, 70 (2012) 344-348.

[56] W. Ahn, K. B. Kim, K. N. Jung, K. H. Shin, C. S. Jin, Synthesis and electrochemical properties of a sulfur-multi walled carbon nanotubes composite as a cathode material for lithium sulfur 
batteries, Journal of Power Sources, 202 (2012) 394-399.

[57] H. L. Wu, L. A. Huff, J. L. Esbenshade, A. A. Gewirth, In Situ EQCM Study Examining Irreversible Changes the Sulfur-Carbon Cathode in Lithium-Sulfur Batteries, ACS Appl Mater Interfaces, 7 (2015) 20820-20828.

[58] K. Amin, Q. Meng, A. Ahmad, M. Cheng, M. Zhang, L. Mao, K. Lu, Z. Wei, A Carbonyl Compound-Based Flexible Cathode with Superior Rate Performance and Cyclic Stability for Flexible Lithium-Ion Batteries, Adv Mater, 30 (2018).

[59] H. Kim, J. Lee, H. Ahn, O. Kim, M. J. Park, Synthesis of three-dimensionally interconnected sulfur-rich polymers for cathode materials of high-rate lithium-sulfur batteries, Nat Commun, 6 (2015) 7278.

[60] X. Li, J. Liang, J. Luo, C. Wang, X. Li, Q. Sun, R. Li, L. Zhang, R. Yang, S. Lu, H. Huang, X. Sun, High-Performance Li-SeSx All-Solid-State Lithium Batteries, Adv Mater, 31 (2019) e1808100.

[61] F. Sun, H. Cheng, J. Chen, N. Zheng, Y. Li, J. Shi, Heteroatomic SenS 8 -n Molecules Confined in Nitrogen-Doped Mesoporous Carbons as Reversible Cathode Materials for High-Performance Lithium Batteries, ACS Nano, 10 (2016) 8289-8298.

[62] J. Zhou, T. Qian, N. Xu, M. Wang, X. Ni, X. Liu, X. Shen, C. Yan, Selenium-Doped Cathodes for Lithium-Organosulfur Batteries with Greatly Improved Volumetric Capacity and Coulombic Efficiency, Adv Mater, 29 (2017). 
2019-10-14

\section{Optimized synthesis of}

ultrahigh-surface-area and

oxygen-doped carbon nanobelts for high cycle-stability lithium-sulfur batteries

Zou, Jizhao

Electrochemical Society

Zou J, Niu Y, Tu W, et al., (2019) Optimized synthesis of ultrahigh-surface-area and oxygen-doped carbon nanobelts for high cycle-stability lithium-sulfur batteries. Journal of The Electrochemical Society, Volume 166, Issue 14, January 2019, pp. A3464-A3473

https://doi.org/10.1149/2.1111914jes

Downloaded from Cranfield Library Services E-Repository 